\title{
Espiritualidade e Formação nos Programas de Residência em Saúde de uma Cidade no Nordeste Brasileiro
}

\section{Spirituality and Training in Health Residency Programs in a Northeastern Brazilian City}

Arturo de Pádua Walfrido Jordán ${ }^{I}$ Leopoldo Nelson Fernandes Barbosa ${ }^{I}$

\section{PALAVRAS-CHAVE}

- Espiritualidade.

- Educação em Saúde.

- Educação Continuada.

- Internato e Residência

\section{RESUMO}

Introdução: Evidências da literatura mostram a influência da espiritualidade na saúde dos indivíduos. No entanto, poucas instituições de ensino em saúde no Brasil incluem a temática em seus conteúdos programáticos. Essa realidade é ainda mais evidente ao tratarmos de programas de especialização ou residência na área da saúde. Objetivo: Analisar a abordagem de conteúdo relacionado à espiritualidade nos programas de residência da Secretaria de Saúde da cidade do Recife (Sesau-Recife). Método: O estudo foi aprovado por Comitê de Ética em pesquisas sob o Parecer no 2.136.503. Trata-se de um estudo exploratório, descritivo, com abordagem combinada, que envolveu a análise dos Projetos Político-Pedagógicos por meio de roteiro de análise documental e entrevistas com residentes e preceptores de oito programas de residência da Sesau-Recife, utilizando um roteiro de entrevista semiestruturada. As informações coletadas foram analisadas segundo a proposta de Minayo. Resultados e discussão: Na análise documental não foi encontrado conteúdo sobre espiritualidade. Nas entrevistas, o conteúdo coletado foi categorizado em: conceitos; espiritualidade baseada em evidências; espiritualidade e cuidado; espiritualidade e finitude; espiritualidade e formação. Na análise foi observado que os preceptores e residentes, em sua maioria, não conhecem os conceitos, as evidências e a aplicabilidade da espiritualidade na prática clínica e na formação em saúde. Os entrevistados demonstraram interesse em capacitação na temática e consideraram relevante a inclusão da espiritualidade no conteúdo dos programas. Conclusão: A abordagem da espiritualidade em cursos de saúde vem crescendo rapidamente no cenário internacional e de forma mais lenta, porém presente, no cenário nacional. Essa realidade inclui de forma mais contundente os cursos de graduação. Ao se tratar de pós-graduação, no entanto, pouco se avançou. Os programas de residência da Sesau-Recife são um exemplo desta assertiva, visto que não contemplam, ao menos de forma oficial, conteúdos de espiritualidade em seus programas. Neste sentido, visto o benefício que traz para os indivíduos, recomenda-se a inclusão da espiritualidade nos conteúdos oficiais desses programas. 


\section{KEY-WORDS}

- Spirituality.

- Health Education.

- Education, Continuing.

- Internship and Residency.

\begin{abstract}
Introduction: Evidence from the literature shows the influence of spirituality on individuals' health. However, few health education institutions in Brazil include the topic in their course programs. This reality is even more evident when we are dealing with specialization or residency programs in the health area. Objective: To analyze how spirituality-related content is approached in the Health Department Residency Programs of the city of Recife (Sesau-Recife). Method: The study was approved by the Research Ethics Committee, report number 2,136,503. It is an exploratory, descriptive study with a combined approach that involved analysis of the Political Pedagogical Projects through a documentary analysis script and interviews with residents and preceptors of 8 Residency Programs in Sesau-Recife, using a semi-structured interview script. Once collected, these were analyzed according to Minayo's proposal. Results and discussion: In the documentary analysis, no content on spirituality was found. In the interviews, the collected content was categorized into: concepts; evidence-based spirituality; spirituality and care; spirituality and finitude; spirituality and training. In the analysis, it was observed that preceptors and residents, for the most part, do not know the concepts, evidence and applicability of spirituality in clinical practice and in health education. The interviewees showed an interest in training in the subject and considered relevant the inclusion of spirituality in the course content. Conclusion: The approach of spirituality in health courses has been growing rapidly in the international setting and more slowly in the domestic sphere. This reality is even more categoric for undergraduate courses. As regards graduate studies, however, little progress has been made. The residency programs of Sesau-Recife are an example of this assertion, since they do not contemplate, at least officially, the contents of spirituality in their programs. In this sense, given the benefit it brings to individuals, it is recommended that spirituality be included in the official contents of these programs.
\end{abstract}

Recebido em: 19/10/18

Aceito em: 6/3/19

\section{INTRODUÇÃO}

Falar sobre espiritualidade ainda é novidade para muitos profissionais de saúde, até porque os bancos de dados nacionais e internacionais contêm uma gama de pesquisas que atribuem conceitos diferentes à espiritualidade, o que dificulta seu entendimento ${ }^{1}$.

Espiritualidade pode ser entendida como a busca pessoal pelo entendimento de respostas a questões sobre a vida, seu significado e relações com o sagrado e transcendente, que pode ou não estar relacionada a propostas de determinada religião².

$\mathrm{Na}$ atualidade, ao tratar de saúde, a $\mathrm{OMS}^{3}$ ratifica a importância da espiritualidade ao referir que o profissional de saúde deve observar os pacientes e seus familiares em quatro aspectos: físico, psíquico, social e espiritual.

Neste contexto, a literatura traz evidências acerca da relação entre saúde e espiritualidade. Koenig ${ }^{12}$, em revisão sistemática, analisou estudos quantitativos de 1872 a 2010 que envolviam religião/espiritualidade e saúde física e mental. A revisão evidenciou uma relação direta da espiritualidade com bem-estar e felicidade, esperança, otimismo e autoestima. No âmbito da saúde mental, mostrou relação inversa com depressão, ansiedade e uso e abuso de substâncias psicoativas".

Outra revisão sistemática que avaliou religião/espiritualidade e doenças cardiovasculares em mais de 3.200 estudos quantitativos encontrados nas maiores bases de dados, como Medline e PsycINFO, evidenciou relação inversa entre espiritualidade e hipertensão arterial sistêmica (HAS), doença arterial coronariana (DAC) e infecções pós-cirúrgicas em cirurgias cardíacas $^{5}$.

Apesar deste corpo de evidências sobre a influência da espiritualidade sobre a saúde, é preciso avançar sob o ponto de vista de formação profissional sobre o tema ${ }^{6}$. Observa-se, por exemplo, que os enfermeiros entendem que faz parte das suas atribuições entender e abordar a espiritualidade dos pacientes, mas a maioria dos profissionais alega falta de treinamento específico 7,8

Neste sentido, a Association of American Medical Colleges (AAMC) elaborou o documento National Competencies in Spirituality and Health for Medical Education (NCSMD), que traça domínios e competências essenciais a serem desenvolvidos 
pelos estudantes sobre espiritualidade, fomentando, assim, a inclusão da temática nos currículos das escolas médicas ${ }^{9,10}$.

Da mesma forma, a Associação Americana de Enfermeiros, a Associação Americana de Faculdades de Enfermagem e a Organização Mundial da Saúde ratificam o cuidado espiritual no Escopo e Padrões de Práticas de Enfermagem oficialmente na Declaração de Política Social dos Enfermeiros, no Código de Ética das Enfermeiras, no 2008 Essentials of Baccalaureate Education e no Código de Ética do Conselho Internacional de Enfermeiros ${ }^{7,11}$.

Neste sentido, um estudo mostra que, em 2010, 40\% das escolas médicas brasileiras possuíam conteúdos relacionados à espiritualidade em seus currículos e 54\% dos entrevistados consideravam muito importante a abordagem desta temática nos currículos dessas escolas ${ }^{12}$.

Nesse contexto, a proposta do presente artigo é trazer analisar os programas de residência em saúde da Sesau-Recife acerca da abordagem da espiritualidade em seus conteúdos práticos e/ou teóricos.

\section{OBJETIVOS}

Analisar a abordagem de conteúdo relacionado à espiritualidade nos programas de residência da Secretaria de Saúde da cidade do Recife; descrever e analisar o conteúdo relacionado à espiritualidade abordado nos Projetos Político-Pedagógicos (PPP) dos programas de residência da Secretaria de Saúde da cidade do Recife; analisar a compreensão dos residentes e preceptores em relação à abordagem do conteúdo relacionado à espiritualidade durante a formação na residência; analisar, na perspectiva dos residentes e preceptores, a relevância e necessidade da abordagem de conteúdo relacionado à espiritualidade durante a residência.

\section{MÉTODO}

Trata-se de um estudo exploratório, descritivo, com abordagem combinada, que envolve pesquisa documental dos Projetos Político-Pedagógicos (PPP) e método qualitativo por meio de entrevistas com dois preceptores e dois residentes de cada um dos oito programas de residência que preencheram os critérios de inclusão do estudo, totalizando 32 sujeitos selecionados por conveniência. Os programas são: Enfermagem em Atendimento Pré-Hospitalar, Enfermagem Obstétrica, Medicina de Família e Comunidade, Médica em Psiquiatria, Odontologia em Saúde Coletiva, Multiprofissional em Vigilância em Saúde, Multiprofissional na Rede de Atenção Psicossocial e Multiprofissional em Saúde Coletiva.

Para a análise documental foi estruturado um roteiro de análise documental e para as entrevistas foi utilizado um ro- teiro de entrevista semiestruturada. A coleta dos dados foi realizada entre dezembro de 2017 e janeiro de 2018 após a aprovação do Comitê de Ética e Pesquisa com Seres Humanos da Faculdade Pernambucana de Saúde, sob o Parecer $n^{\circ} 2.136 .503$. Os sujeitos que aceitaram participar do estudo assinaram um Termo de Consentimento Livre e Esclarecido que continha todos os dados relevantes da pesquisa. Todas as entrevistas foram gravadas, transcritas e analisadas por meio da análise de conteúdo, tal como proposto por Minayo.

Foram seguidos os preceitos das Resoluções 466/12 e 510/16 do Conselho Nacional de Saúde, visando à preservação dos quatro referenciais da bioética: autonomia, não maleficência, beneficência e justiça.

\section{RESULTADOS E DISCUSSÃO}

\section{Análise dos Projetos Político-Pedagógicos (PPP)}

Os PPP são considerados projetos porque trazem a ideia de futuro a partir do presente, o projetar; são políticos porque levam a uma direção, a um objetivo final; e são pedagógicos porque se baseiam na construção coletiva com todos os envolvidos no processo da instituição, levando a práticas sociais emancipatórias, com a formação de um sujeito social crítico, solidário, compromissado, criativo e participativo. Devem englobar todas as dimensões da vida na instituição: infraestrutura física e equipamentos (salas, equipamentos e aspectos financeiros); organização e gestão da prática pedagógica (como avaliação); gestão democrática da escola (como democratização das informações); formação e condições de trabalho dos professores (como carga horária específica e corpo docente qualificado) e ações de apoio aos estudantes (como acesso aos equipamentos da instituição para sua formação) $)^{13,14}$.

Neste sentido, a análise documental dos oito programas de residência incluídos neste estudo demonstrou que apenas o Programa de Enfermagem em Atendimento Pré-Hospitalar possui um documento oficial nos moldes de um PPP.

O programa não inclui a temática "espiritualidade" de forma direta, porém na descrição do PPP podemos ver possibilidades para a sua abordagem.

O perfil do egresso descreve que o profissional deve ser habilitado em uma visão holística, o que nos remete à visão integral que o profissional deve ter a respeito dos usuários do sistema. Sendo uma dimensão de cada ser humano, a espiritualidade certamente faz parte da abordagem integral ${ }^{15}$. Esse perfil também descreve que o profissional deve aprender a refletir, a discutir a prática de saúde baseada em evidências utilizando protocolos e diretrizes, sem desconsiderar o cenário loco-regional. A literatura traz várias evidências da influência da espiritualidade na saúde do indivíduo ${ }^{4,5}$. 
Os objetivos gerais do curso incluem: formar enfermeiros especialistas na área de concentração, com visão humanista, reflexiva e crítica, qualificados para atuar no atendimento integral ao paciente. Este objetivo mostra claramente a necessidade de humanização. Desta forma, abordar espiritualidade também leva à humanização, visto que ela se revela pelo amor, pela sensibilidade, pela compaixão, pela escuta do outro, pela responsabilidade e pelo cuidado como atitude fundamental ${ }^{15}$.

Em relação às entrevistas, dos 32 sujeitos entrevistados, a maioria é do sexo feminino, solteira, sem filhos, com média de 33 anos de idade, renda média de $\mathrm{R} \$$ 6.171,00 e a maioria declarando ter religião e ser da religião católica.

As falas foram divididas em cinco temas: Conceitos, Espiritualidade Baseada em Evidências, Espiritualidade e Cuidado, Espiritualidade e Finitude, e Espiritualidade e Formação.

A categoria Conceitos foi subdividida em duas subcategorias: (a) espiritualidade, religião, religiosidade e Deus; (b) espiritualidade, transcendência e fé.

Na subcategoria espiritualidade, religião, religiosidade e Deus, destacam-se as seguintes falas:

Espiritualidade para mim é, a questão da religião... independentemente de você seguir uma religião ou não, mas você seguir, vamos dizer assim, conceitos corretos. (R2)

é o meu relacionamento com Deus, espiritualidade (R5)

Falar sobre espiritualidade ainda é novidade para muitos. Os bancos de dados nacionais e internacionais contêm uma gama de pesquisas que atribui conceitos diferentes à espiritualidade, dificultando o entendimento dos termos e, em alguns casos, levando à falsa interpretação de que os resultados obtidos por determinadas pesquisas podem ser estendidos a toda a população, quando na verdade são aplicáveis apenas a determinado grupo ${ }^{1}$.

Espiritualidade não é religião, religiosidade ou Deus, porém ter e praticar uma religião, assim como acreditar e buscar a Deus podem ser um caminho para a espiritualidade ${ }^{2,9}$.

As seguintes falas se enquadram em espiritualidade, transcendência e fé:

Espiritualidade eu entendo como a parte da fé que a pessoa tem em acreditar. (P13)

espiritualidade é um estado de conexão com algo que é, que vai além do físico e do corpo, da mente, que é um estado de transcendência mesmo. Onde expansão de consciência é algo muito maior, que não é muito desse mundo material. (P8)
É interessante destacar nestas falas que os entrevistados usam termos como expansão da consciência, algo além, transcendência e fé para definir espiritualidade. Apesar de não conhecerem de fato o conceito, os entrevistados se aproximam dele:

Espiritualidade é uma busca pessoal pelo entendimento de respostas a questões sobre a vida, seu significado e relações com o sagrado e transcendente, que pode ou não estar relacionada a propostas de determinada religião ${ }^{2}$, assim como pode ser expressa mediante a participação de grupos religiosos que possuem algo em comum, como fé em Deus, naturalismo, humanismo, família e arte ${ }^{16}$.

A categoria Espiritualidade Baseada em Evidências foi subdividida em duas subcategorias: (a) espiritualidade e promoção e prevenção à saúde; (b) espiritualidade e tratamento de enfermidades.

Ao tratar de espiritualidade como fator promotor e de prevenção à saúde, destacam-se as seguintes falas:

Gente com sindrome de burnout, alguns funcionários inclusive desenvolvendo sindrome do pânico, uma série de situações, eu acredito que por justamente não serem trabalhadas essas questões (espirituais). (P1)

É relevante sim (trabalhar espiritualidade) porque a gente já tem inúmeras pesquisas científicas comprovando que os pacientes com espiritualidade têm um fator de proteção contra doença mental. Quando desenvolvem a doença mental, costumam recuperar-se mais rapidamente, têm menores indices de suicídio, enfim... (R10)

$\mathrm{Na}$ atualidade, os estudos relacionados à espiritualidade vêm tendo um incremento em publicações relevantes em bancos de dados internacionais. Lucchetti e Lucchetti ${ }^{17}$, por exemplo, afirmam que houve um aumento de 1.547 artigos publicados anualmente em 1999 para 2.750 artigos publicados anualmente em 2013 na base Pubmed.

Ao tratar de promoção à saúde, estudos mostram que a espiritualidade tem relação direta com bem-estar, felicidade, esperança, otimismo, autoestima e melhoria da qualidade de vida, além de diminuir o hábito de fumar e o uso e abuso de substâncias psicoativas, bem como apresenta uma relação inversa com depressão, ansiedade e tentativas de suicídio ${ }^{4,5}$.

Ilustrando estes dados, em estudo com 1.154 estudantes de 13 a 19 anos no município de Olinda em Pernambuco ao avaliarem o uso de drogas ilícitas e binge drinking, Cipriano et al. ${ }^{18}$ afirmam que não ter afiliação religiosa aumentou em 37\% a prevalência de usar drogas em comparação aos estudantes que in- 
formaram ter religião. Isso, de acordo com os autores, corrobora o resultado de outros estudos que tratam a religião como fator que protege contra o uso de drogas, possivelmente em razão das normas de conduta disseminadas pela maioria das religiões.

Os próximos entrevistados trazem como possibilidade a abordagem da espiritualidade no tratamento de enfermidades:

durante os momentos de prática, as pacientes costumam dizer que não vão aguentar aquele momento, que é insuportável $e$, assim, vários preceptores, inclusive a gente, residente, costuma abordá-las dizendo que é normal, que é fisiológico, que ela converse com Deus... (R4)

leva muito em consideração a religião como um processo terapêutico também... o paciente de saúde mental traz consigo muito discurso religioso... E aí às vezes nas intervenções a gente acaba usando a espiritualidade como terapêtico. (R7)

Estudos demonstram que espiritualidade tem relação inversa com ansiedade, depressão, uso e abuso de substâncias psicoativas, HAS, DAC e infecções pós cirúrgicas ${ }^{4,5}$.

Uma revisão sistemática com metanálise ${ }^{19}$ que investigou o impacto das intervenções em saúde e espiritualidade com base em 4.751 estudos clínicos randomizados encontrados em bancos de dados como PubMed, Scopus, Web of Science, PsycINFO, Cochrane Collaboration, Embase e SciELO evidenciou diminuição de sintomas clínicos ligados à ansiedade.

Da mesma forma, outra revisão sistemática com metanálise $\left(\mathrm{Oh}\right.$ e $\left.\mathrm{Kim}^{20}\right)$ que avaliou a efetividade da intervenção espiritual biologicamente, psicologicamente e espiritualmente em um total geral de 2.522 estudos pesquisados nas bases de dados eletrônicas mostrou resultados moderados ao se intervir na espiritualidade de pacientes com depressão e ansiedade ${ }^{20}$.

A categoria Espiritualidade e Cuidado não teve subdivisão. Os entrevistados afirmaram que:

Então, eu acho que, de tudo que se citou aí, eu destacaria a questão do cuidado, a presença desse amor, esse sentido, esse fazer. (P11)

Então, acredito que sim (A importância de abordar espiritualidade), de acordo com vários aspectos desse (conceito de espiritualidade), a gente tem trazido, tanto em relação ao trato com os residentes, como ao cuidado com o ser humano, e quanto ao trato com a equipe multidisciplinar. (P2)

Todas as profissões da área da saúde têm como pressuposto básico o cuidado. Cuidar deve partir da reflexão na ação, mergulhando, assim, em todo o processo de cuidado do indivíduo, desde o pré-cuidado até a continuidade desse cuidado, o que, em última instância, favorece a singularidade desse processo ${ }^{21}$.

Esta reflexão vai ao encontro das ideias de Boff ${ }^{15}$, para quem a espiritualidade se manifesta pelo amor, pela sensibilidade, pela compaixão, pela escuta do outro, pela responsabilidade e pelo cuidado como atitude fundamental. Neste sentido, os enfermeiros, por exemplo, entendem o cuidado espiritual como parte intrínseca do cuidado em enfermagem ${ }^{7,8}$.

A categoria Espiritualidade e Finitude foi subdividida em duas subcategorias: (a) espiritualidade, morte e o morrer; (b) espiritualidade e proteção ao suicídio.

No que concerne a espiritualidade, morte e o morrer, destacam-se as seguintes falas:

As situações críticas que acontecem (risco de morte do paciente), em que a gente precisa estar lá do lado daquela pessoa e fortalecer e trazer um pouco de esperança, de discutir certas situações que acontecem e poder realmente fortalecer e ajudar o outro. (P2)

alguém que confia em Deus, que aí até quando está próximo da morte, você até tenha uma aceitação, eu acho até mais tranquilo, não você na morte, mas com sua família, com alguém familiar. Você, quando tem essa espiritualidade, você aceita mais, se conforta. (P4)

A morte é vista como algo medonho, algo inconcebível de acontecer conosco. Em caso de enfermidades graves, esse contexto se agrava, pois a pessoa perde a autonomia ao não opinar sobre o seu tratamento, feito exclusivamente pelos profissionais de saúde, com a consequente transformação do sujeito em objeto ${ }^{22}$. E estes profissionais não estão preparados para lidar com a finitude, pois são treinados para ver a morte sem alma, não levando em conta as interações subjetivas que ocorrem com os pacientes ${ }^{23}$.

Quando se trata, por exemplo, de pacientes com câncer, ficam evidentes os anseios gerados em relação à finitude, não só para os pacientes, mas também para seus familiares, pois, afinal, a doença significa uma ameaça à vida. Essa questão também afeta os profissionais de saúde, pois eles foram treinados para evitar a morte, e a "tentação tecnológica" pode surgir, induzindo-os a fragmentar o indivíduo, esquecendo de observá-lo em sua integralidade, e a não levar em consideração os anseios, sofrimentos e a espiritualidade do paciente ${ }^{24}$.

Estudar e praticar espiritualidade, além de prevenirem as consequências geradas nos profissionais de saúde que lidam 
com questões de morte, ainda fornecem subsídios para esses mesmos profissionais poderem estimular questões como esperança, fé, bem-estar, felicidade, otimismo e autoestima ${ }^{4,24}$.

Em relação a espiritualidade e proteção ao suicídio, destacam-se as seguintes falas:

Acredito que é um tema (espiritualidade) que precisa realmente ser mais bem abordado, mais bem trabalhado, porque nós estamos vivendo um período extremamente difícil... eu acho que cabe aqui a gente falar do aumento do número de suicídios. (P1)

Então, por exemplo, na residência de Psiquiatria a gente lida com pessoas que estão pensando em tirar a vida, pessoas que apresentam doenças que não vão melhorar, estão crônicas. $O$ que elas precisam, muitas vezes, é falar de outros aspectos que não somente o agora. (P9)

Houve um aumento de casos de suicídio de $60 \%$ em todo o mundo nos últimos 45 anos, a ponto de atualmente o suicídio ser, em muitos países, a segunda ou terceira causa de morte dependendo da faixa etária. A cada ano, cerca de um milhão de pessoas morre devido ao suicídio, o que representa uma morte a cada 40 segundos. No Brasil, foram registrados oficialmente 8.550 suicídios em 2005 e atualmente acontecem 24 mortes por suicídio por dia, isto é, uma morte por hora ${ }^{25}$.

Os índices de suicídio têm aumentado também entre os profissionais de saúde, como médicos e enfermeiros, devido, entre outras causas, ao ambiente de trabalho estressante e ao envolvimento constante com situações de sofrimento e morte ${ }^{26}$.

Um recente estudo (Costa e Souza ${ }^{27}$ ) que investigou a vida de oito idosos que cometeram suicídio no Amazonas evidenciou, por meio de entrevistas com familiares, que as perdas, de diferentes matizes, incluindo morte de familiares, diagnóstico de doenças crônicas, afastamento do trabalho, baixa autoestima, conflitos familiares e uso de drogas psicoativas, foram consideradas as causas do desfecho. Os autores desse estudo recomendam melhor qualificação dos profissionais para abordarem diagnósticos de doenças crônicas e incuráveis, levando sempre em consideração os fatores socioculturais e psicológicos dos envolvidos, além de empoderar os idosos por meio de estratégias intersetoriais que visem a melhor resiliência ao lidar com perdas e conflitos familiares ${ }^{27}$.

Nesse contexto, estudos mostram que espiritualidade é um fator que protege contra o suicídio, por ter correlação com aqueles matizes apresentados ao proporcionar otimismo, melhor autoestima, melhor qualidade de vida, melhora de doenças crônicas e ainda diminuir a ansiedade e a depressão ${ }^{4,5}$.
A categoria Espiritualidade e Formação foi subdividida em duas subcategorias: (a) ideias preconcebidas; (b) espiritualidade na formação e integralidade.

No que concerne à existência de ideias preconcebidas acerca da espiritualidade, destacam-se:

eu vi um pouquinho na disciplina de políticas públicas... a maioria dos alunos ficou meio receosa com aquilo, tipo assim: "como assim, a professora está falando sobre espiritualidade numa disciplina que é de formação de profissionais de saúde?" (R15)

Porque algumas pessoas têm a visão da espiritualidade enquanto religião apenas, uma limitação do credo religioso. Então, sabendo que vai para além disso, talvez na abordagem ela tenha que tomar esse cuidado. (R13)

Profissionais de saúde alegam, como dificuldade para abordar espiritualidade com seus pacientes: falta de conhecimento sobre o assunto, falta de treinamento, falta de tempo, desconforto com o tema, medo de impor pontos de vista religiosos ao paciente, entendimento de que o conhecimento da religião não é relevante para o tratamento, assim como a opinião de que isso não faz parte do seu papel enquanto profissional - ideias preconcebidas, que precisam ser removidas para a vulgarização desta abordagem de forma segura ${ }^{28}$.

Um passo importante que certamente contribuiu para a quebra dos preconceitos acerca da temática "espiritualidade" foi a publicação em 1994, pela Associação Americana de Psiquiatria, do Manual de Diagnóstico e Estatística dos Transtornos Mentais - Quarta Edição (DSM-IV). Esse manual incluiu "Problemas espirituais e religiosos" como instância diagnóstica, que, segundo Almeida ${ }^{29}$, foi introduzida "para direcionar a atenção clínica, justificando a avaliação de experiências religiosas e espirituais como parte constituinte da investigação psiquiátrica sem necessariamente julgá-las como psicopatológicas." ${ }^{29}$

Outro passo importante partiu da Organização Mundial da Saúde (OMS) ao incluir em seus manuais de cuidados paliativos que o profissional de saúde deve observar os pacientes e ainda seus familiares, ao abordar dor ou outros problemas, em quatro aspectos: físico, psíquico, social e espiritual ${ }^{3}$.

Ao tratar de espiritualidade na formação e integralidade, foram coletadas as seguintes falas:

A relevância (em abordar espiritualidade) é extremamente importante porque faz a gente refletir inclusive sobre as práticas e o que é que a gente pode reabordar no conteúdo da residência no sentido de trabalhar mais esse lado espiritual, que muitas vezes é esquecido. (P3) 
(Pensando em uma) residência que lida com o cuidado integral das pessoas e não vê só a doença, eu acho que a espiritualidade está em todos os momentos da nossa residência, do nosso cuidado. (P7)

A integralidade faz parte dos princípios do Sistema Único de Saúde (SUS. Ela surgiu a partir de concepções da saúde coletiva com a tentativa de inclusão principalmente no que concerne à atenção primária. Pode ser entendida de três formas: uma prática sanitária individual, incluindo fatores psicológicos e sociais implicados na determinação das doenças; uma forma de organização dos serviços com uma mescla da visão assistencial com a preventiva; uma prática de políticas específicas ou especiais, onde estariam ações voltadas para resolver problemas específicos em segmentos populacionais bem definidos ${ }^{30}$. Neste sentido, a espiritualidade tem espaço junto à integralidade principalmente no que se refere à prática sanitária individual, na medida em que incorpora o cuidado espiritual a este processo ${ }^{7}$.

Em relação à formação profissional em espiritualidade, percebe-se, pelas falas dos entrevistados, que é necessário investir mais. Neste contexto, a literatura mostra uma gama de possibilidades para sua abordagem: palestras, discussões em pequenos grupos, entrevistas padronizadas de pacientes, acompanhamento de capelães e leituras específicas, disciplinas optativas, extensão universitária e por meio da atuação de grupos de estudos e/ou ligas acadêmicas ${ }^{31}$.

\section{CONCLUSÃO}

Programas de residência são considerados "padrão ouro" da pós-graduação lato sensu para a formação de especialistas. Assim, devem proporcionar uma formação que inclua em seus conteúdos e práticas tudo o que for relevante para cumprir sua missão, sempre levando em conta as melhores evidências disponíveis.

Embora a literatura nacional e internacional evidencie que a espiritualidade traz benefícios à saúde dos indivíduos e por isso estimule a formação nessa temática, o presente estudo demonstrou que os programas de residência em Saúde da Secretaria de Saúde do Recife não o fazem, ao menos de forma oficial.

Muitos dos entrevistados abordam preceitos de espiritualidade com os pacientes, porém sem associar a prática ao conceito. Pode-se destacar neste ponto o cuidado, a empatia, o respeito e o estímulo à busca por significado e propósito para a vida. É importante destacar que, ao conhecerem o significado de espiritualidade durante o estudo, os entrevistados reconheceram a relevância e a necessidade da abordagem desse conteúdo nos programas, como também mostraram interesse na capacitação do tema para sua própria qualificação.

Desta forma, após a análise de todos os dados contidos neste trabalho, com a convicção da necessidade de formação em espiritualidade tanto para preceptores como para residentes, os autores sugerem a inclusão da espiritualidade nos conteúdos e práticas dos programas de residência em saúde.

\section{REFERÊNCIAS}

1. Moberg DO. Assessing and measuring spirituality: Confronting dilemmas of universal and particular evaluative criteria [Internet]. Vol. 9, Journal of Adult Development. Kluwer Academic Publishers-Plenum Publishers; 2002 [cited 2018 Mar 10]. p. 47-60. Available from: http:/ /link. springer.com/10.1023/A:1013877201375

2. Koenig HG, McCullough ME, Larson DB.Handbook of Religion and Health. 2001 | Online Research Library: Questia [Internet]. Available from: https://www.questia.com/ read/106205934/handbook-of-religion-and-health

3. World Heath Organization. Cancer Control Knowledge into Action - WHO Guide for Effective Programmes Palliative Care. 2007 [cited 2016 Nov 6];51. Available from: http:/ / www.who.int/cancer/media/FINAL-PalliativeCareModule.pdf

4. Koenig HG. Religion, Spirituality, and Health: The Research and Clinical Implications. ISRN Psychiatry [Internet]. 2012 [cited 2016 Nov 6];2012:1-33. Available from: http:/ / www.ncbi.nlm.nih.gov/pubmed/23762764

5. Lucchese, F. A., \& Koenig, H. G. (2013). Religion, spirituality and cardiovascular disease: research, clinical implications, and opportunities in Brazil. Revista Brasileira de Cirurgia Cardiovascular, 28(1), 103-128. https://doi. org/10.5935/1678-9741.20130015

6. Moreira-Almeida, A. (2013). Religion and health: The more we know the more we need to know. World Psychiatry : Official Journal of the World Psychiatric Association (WPA), 12(1), 37-38. https:/ / doi.org/10.1002/wps.20009

7. Bennett V, Thompson ML. Teaching spirituality to student nurses. J Nurs Educ Pract [Internet]. 2014 Nov 13 [cited 2018 May 1];5(2):26. Available from: http://www.sciedu. ca/journal/index.php/jnep/article/view/5105/3523

8. Pike J. Spirituality in nursing: A systematic review of the literature from 2006-10. Br J Nurs. 2011;20:743-9.

9. Association of American Medical Colleges. Report III Contemporary Issues in Medicine: Communication in Medicine [Internet]. 1999 [cited 2016 Nov 9]. Available from: members.aamc.org/eweb/upload/ContemporaryIssuesMedCommunMedicineReportIII.pdf 
10. Puchalski CM, Blatt B, Kogan M, Butler A. Spirituality and health: the development of a field. Acad Med [Internet]. 2014 Jan [cited 2016 Nov 1];89(1):10-6. Available from: http:/ / www.ncbi.nlm.nih.gov/pubmed/24280839

11. American Association of Colleges of Nursing (AACN). The Essentials of Baccalaureate Education for Professional Nursing Practice [Internet]. 1st ed. Washington, DC; 2008 [cited 2018 May 1]. 63 p. Available from: http://www. aacnnursing.org/Portals / 42/Publications / BaccEssentials08.pdf

12. Koenig HG. Medicina, religião e saúde - O Encontro da Ciência e da Espiritualidade [Internet]. 1st ed. Porto Alegre: L\&PM Editores; 2012 [cited 2018 Mar 20]. 236 p. Available from: http://www.lpm.com.br/site/default. asp? Template $=$. . /livros /layout_produto.asp\&CategoriaI $\mathrm{D}=615261 \& \mathrm{ID}=492220$

13. Ministério da Educação e Cultura. Projeto Político-Pedagógico: dimensões conceituais [Internet]. Escola de Gestores da Educação Básica. [cited 2018 May 30]. Available from: http:/ / escoladegestores.mec.gov.br/site/2-sala_projeto_ vivencial/pdf/dimensoesconceituais.pdf

14. Ministério da Educação e Cultura. Projeto Político-Pedagógico: dimensões metodologicas [Internet]. Escola de Gestores da Educação Básica. [cited 2018 May 30]. Available from: http: / / escoladegestores.mec.gov.br/site/2-sala_ projeto_vivencial/pdf/dimensoesconceituais.pdf

15. Boff L. Espiritualidade: um caminho de transformação, Volume 1 [Internet]. Sextante; 2001 [cited 2016 Nov 16]. 94 p. Available from: https://books.google.com/ books?id=141ZAAAAMAAJ\&pgis $=1$

16. Puchalski C. Task force report: spirituality, cultural issues, and end of life care. in: Association of American Medical Colleges report III: contemporary issues in medicine: communication in medicine, medical school objectives project October 1999 (MSOP III). Assoc Am Med Coll. 1999;(Washington, DC):25-26.

17. Lucchetti G, Lucchetti ALG. Spirituality, Religion, and Health: Over the Last 15 Years of Field Research (1999-2013). Int J Psychiatry Med [Internet]. 2014 Jan 1 [cited 2016 Nov 1];48(3):199-215. Available from: http://ijp.sagepub.com/ lookup/doi/10.2190/PM.48.3.

18. Cipriano J, Carolina A, Costa DQ, Melo PA De. Uso de drogas ilícitas e binge drinking entre estudantes adolescentes. Rev Saude Publica [Internet]. 2017 [cited 2018 Mar 17];51(83):1-7. Available from: http://www.rsp.fsp. usp.br/wp-content/uploads/articles_xml/0034-8910rsp-S1518-87872017051006863/0034-8910-rsp-S151887872017051006863-pt.x83902.pdf
19. Gonçalves, J. P. B., Lucchetti, G., Menezes, P. R., \& Vallada, H. (2015). Religious and spiritual interventions in mental health care: a systematic review and meta-analysis of randomized controlled clinical trials. Psychological Medicine, 45(14), 2937-2949. https://doi.org/10.1017/ S0033291715001166

20. Oh, P.-J., \& Kim, Y.-H. (2012). Meta-Analysis of Spiritual Intervention Studies on Biological, Psychological, and Spiritual Outcomes. Journal of Korean Academy of Nursing, 42(6), 833. https: / / doi.org/10.4040/jkan.2012.42.6.833

21. Waldow V. Momento de cuidar: momento de reflexão na ação. Revista Brasileira de Enfermagem [Internet]. 2008 Feb [cited 2018 Mar 17];2(1):140-5. Available from: http:/ / www.scielo.br/scielo.php?script=sci_arttext\&pid=S0034$-71672009000100022 \& \operatorname{lng}=$ pt\&tlng=pt

22. Kübler-Ross E. Sobre A Morte e o Morrer [Internet]. 10th ed. Rio de Janeiro: Editora Martins Fontes; 2017 [cited 2018 May 17]. 304 p. Available from: https://www.saraiva.com.br/sobre-a-morte-e-o-morrer-10-ed-2017-9753631. html

23. Silva, G. S. N. (2006). A construção do "ser médico" e a morte: significados e implicações para a humanização do cuidado. Tese de Doutorado, Faculdade de Medicina, Universidade de São Paulo, São Paulo.

24. Espíndula JA, Ranier E, Do M, Bello AA. Religião e espiritualidade : um olhar de profissionais de saúde. Rev Lat Am Enfermagem [Internet]. 2010 [cited 2018 Mar 11];18(6):1-8. Available from: www.eerp.usp.br/rlae

25. Braga LDL, Dell'Aglio DD. Suicídio na adolescência: fatores de risco, depressão e gênero. Contexto Clínicos [Internet]. 2013 Apr 1 [cited 2018 May 16];6(1):2-14. Available from: http://revistas.unisinos.br/index.php/contextosclinicos/article/view/3592

26. Barbosa Freitas AP, Oliveira Abreu AC, Batista Côelho M, Castro Peres T, Laterza Alves IDO. O Fenômeno Do Suicídio Entre Profissionais Da Saúde: Uma revisão bibliográfica. [cited 2018 May 16];1-10. Available from: https:/ / semanaacademica.org.br/system/files/artigos/suicidio_ em_colaboradoes_da_saude.pdf

27. Costa ALS da, Souza MLP de. Narrativas de familiares sobre o suicídio de idosos em uma metrópole amazônica. Rev Saude Publica [Internet]. 2017 [cited 2018 Mar 17];51:121. Available from: http:/ / www.rsp.fsp.usp.br/

28. Lucchetti G, Granero AL, Bassi RM, Latorraca R, Aparecida S. Espiritualidade na prática clínica : o que o clínico deve saber ?*. Rev Bras Clin Medica [Internet]. 2010 [cited 2018 Mar 4];8(2):154-8. Available from: http://files.bvs. br/upload/S/1679-1010/2010/v8n2/a012.pdf 
29. Moreira-Almeida A, Cardeña E. Diagnóstico diferencial entre experiências espirituais e psicóticas não patológicas e transtornos mentais: Uma contribuição de estudos latino-americanos para o CID-11. Rev Bras Psiquiatr [Internet]. 2011 [cited 2018 Mar 10];33(SUPPL.1):521-8. Available from: http:/ / www.scielo.br/pdf/rbp/v33s1/04.pdf

30. Ribeiro de Moraes D. Revisitando As Concepções de Integralidade. 2006 [cited 2018 Mar 18];(1):64-72. Available from: http:/ / www.ufjf.br/nates/files/2009/12/Integralidade.pdf

31. Fortin AH, Barnett KG, ML G, ML G, J G-P, CM P, et al. Medical School Curricula in Spirituality and Medicine. JAMA J Am Med Assoc [Internet]. 2004 Jun 16 [cited 2016 Nov 1];291(23):2883-2883. Available from: http://jama.jamanetwork.com/article.aspx?articleid=198943

\section{CONTRIBUIÇÃO DOS AUTORES}

Ambos os autores contribuíram substancialmente para a concepção, planejamento, análise, interpretação dos dados, elaboração do rascunho, revisão crítica do conteúdo e aprovação da versão final do manuscrito.

\section{CONFLITO DE INTERESSES}

Nenhum dos dois autores deste trabalho possui conflitos de interesse.

\section{ENDEREÇO PARA CORRESPONDÊNCIA}

Rua Aviador Severiano Lins, número 105, Bloco B, Apt 501, Boa viagem, Recife, Pernambuco, Brasil, CEP: 51020-060 